\title{
Evaluation of cutaneous side-effects associated with chemotherapeutic use in oncological patients
}

\author{
Zeynep Utlu1, Handan Bilen² \\ ${ }^{1}$ Department of Dermatology, Erzurum Region Education and Research Hospital, Erzurum, Turkey \\ ${ }^{2}$ Department of Dermatology, Faculty of Medicine, Ataturk University, Erzurum, Turkey \\ Adv Dermatol Allergol 2021; XXXVIII (6): 1078-1085 \\ DOI: https://doi.org/10.5114/ada.2020.99943
}

\begin{abstract}
Introduction: Side-effects are frequently encountered in classic chemotherapy. However, the recent development of targeted treatments has resulted in a diminution of these. The most common side-effects are dermatological. Aim: To investigate cutaneous changes occurring in patients using classic and targeted chemotherapeutic agents, and the prevalence of these changes in the two groups.

Material and methods: One hundred twenty-eight volunteer oncological patients using chemotherapeutic agents were included in this prospective study. Two subgroups were established, patients using classic and those using targeted chemotherapeutic agents.

Results: Xerosis was the most common side-effect, being seen in 93 (72.7\%) of the 128 patients. Other common side-effects included alopecia, pruritus, mucositis, skin pigmentation, and palmar-plantar erythema. The most common side-effects in the classic chemotherapeutic group were xerosis seen in $71(75.5 \%)$ patients, pruritus in 50 (53.2\%), alopecia in 49 (52.1\%), and nail changes in 43 (45.7\%). The most common side-effects in the targeted chemotherapeutic group were xerosis seen in 22 (64.7\%) patients, nail changes in 17 (50\%), alopecia in 15 (44.1\%), and pruritus in 13 (38.2\%).

Conclusions: The most common cutaneous side-effects were less prevalent in the patient group using targeted chemotherapeutic agents than in the classic group. Various side-effects associated with chemotherapeutic use which had not been previously reported were also identified in this study. Classic chemotherapeutic agents caused more serious side-effects requiring discontinuation of treatment than targeted chemotherapeutic agents.
\end{abstract}

Key words: cutaneous side-effects, classic and targeted chemotherapeutic agents.

\section{Introduction}

In the most basic definition, cancer represents unchecked cell growth and division. The disease results in severe morbidity and mortality [1]. Cancer is a growing worldwide health problem. Figures show that 14.1 million new cases were seen worldwide in 2012, with 8.2 million cancer-related deaths being reported. If the increase in cancer diagnoses continues, it is estimated that the annual number of new cases will reach 22 million by 2030 [2]. Cancer is the second most commonly reported cause of death in Turkey after cardiovascular diseases [3].

The growing prevalence of cancer and the high mortality it causes have necessitated greater research into protection against the disease, early diagnosis, and treatment. The essential aim of the treatment of cancer is the complete eradication of the disease. However, since fac- tors such as the stage of the disease, the nature of the tumour, and metastasis make complete elimination difficult, new therapeutic methods have been developed to bring the disease under control, increase the patient's quality of life, and extend survival. Surgery, chemotherapy, radiotherapy, bone marrow transplantation, immunotherapy, hormonal therapies, and alternative medicine techniques are frequently applied in cancer treatment. Primary protection methods are the subject of the greatest focus in treatment, while early diagnosis and surgery are the most important factors in terms of improving the possibility of treatment $[4,5]$.

Chemotherapy is a medical technique employing drugs aimed at halting unchecked cell division and growth, and at inducing apoptosis. A positive relationship has been determined between easy access to these drugs, known as antineoplastics, and survival. Chemo-

Address for correspondence: Assist. Prof. Handan Bilen MD, Department of Dermatology, Faculty of Medicine, Ataturk University, Erzurum, Turkey, phone: +90 44234469 71, fax: +90 44234465 28, e-mail: handanbilen@atauni.edu.tr Received: 25.07.2020, accepted: 25.08.2020. 
therapy has, therefore, become an indispensable element of cancer treatment [6].

Since classic chemotherapeutics suppress mitosis in healthy tissues, they result in more side-effects in regions with greater mitotic activity, such as skin, hair, and bone. These side-effects have led clinicians to investigate novel therapeutic techniques and the development of targeted agents with more specific effects $[4,5]$.

Targeted agents particularly target inter- and intracellular signalling pathways (EGFR, HER2, HER3, HER4, FLT3, RAS, RAF, MEK KIT, RET, MTOR, SRC, EPH, and SCF) [4, 5, 7]. In addition to signalling pathways, these agents also target genes associated with tumour angiogenesis (VEGFR \& TIE2) and the tumour microenvironment (PDGFR \& FGFR) [7]. Although diminished side-effects are observed with treatments involving these agents, they have not been entirely eradicated [5, 7].

The relationship between cytotoxic chemotherapeutic agents and cutaneous side-effects has been investigated and described in detail in recent years. The frequency and spectrum of side-effects have both increased in patients with cancer with the entry into the use of targeted chemotherapeutic drugs, and specific cutaneous side-effects have been described. The identification of cutaneous side-effects associated with epidermal growth factor inhibitors and multikinase inhibitors is a particularly recent phenomenon. Strategies have been introduced which involve reducing the therapeutic dose or suspending or concluding treatment depending on the severity of side-effects. However, predicting cutaneous side-effects at the beginning of treatment is of particular importance in patient management [5].

\section{Aim}

The study aimed to compare the classification and frequency of cutaneous side-effects emerging in association with systemically administered classic and targeted chemotherapeutic agents and to determine new specific cutaneous side-effects.

\section{Material and methods}

\section{Patients and study design}

This prospective study involved patients aged 18 or over referred to the Ataturk University Medical Faculty Dermatology Department, Turkey, and using chemotherapeutics between April and December 2016. All patients were informed about the study before commencement, after which written consent was obtained. Approval for the study was granted by the Ataturk University Medical Faculty Clinical Research Ethical Committee (No. B.30.2.ATA.0.01.00/ dated 28.04.2016).

The study involved 128 randomly selected patients agreeing to take part. Patients' sociodemographic characteristics were recorded. Histories were taken, clinical ex- aminations were performed, and cutaneous side-effects were photographed. Dermatological examinations were performed and recorded on a standardized questionnaire on the week in which mucocutaneous findings were observed after chemotherapy. Two groups were established based on the medications employed - one consisting of patients using classic chemotherapeutic agents, and another of those using targeted chemotherapeutic agents. Classic chemotherapeutics were divided into seven subgroups - alkylating, antimetabolite, alkaloid, topoisomerase inhibitors, anthracyclines, platinum compounds, and hormonal compounds. Targeted chemotherapeutic agents were divided into five subgroups - EGFR inhibitors, BRAF and MEK inhibitors, angiogenesis inhibitors, HER2/neu inhibitors, and proteasome inhibitors. Since none of our patients were using other group drugs, no group was established for these. Patients developing mucocutaneous findings associated with internal malignancy, with mucocutaneous symptoms at the beginning of chemotherapy, and patients receiving radiotherapy were excluded. Dose modification and symptomatic treatments were applied depending on the severity of mucocutaneous side-effects, as suggested in the previous literature [8, 9].

\section{Statistical analysis}

The study data were analysed using SPSS 16.0 software. Compatibility with normal distribution was determined using the Kolmogorov-Smirnov test. The $t$-test was applied for non-normally distributed data in independent groups. The $\chi^{2}$ test at a 95\% confidence interval and a significance level of $p<0.05$ were applied for the comparison of categorical data.

\section{Results}

Sixty-two (48.4\%) of the 128 patients enrolled were men and 66 (51.6\%) were women. The youngest patient was 18 and the oldest 81, and the patients' mean age was 53.85. The mean age of the male patients was 57.69, and that of the female patients was 50.24.

Forty different drugs were used in the patient group, 27 classic chemotherapeutics, and 13 targeted chemotherapeutics. Ninety-four (73.4\%) patients used classic chemotherapeutics and 34 (26.6\%) targeted chemotherapeutics. Twenty-nine (30.9\%) patients in the classic chemotherapeutic group were using antimetabolites, 27 (28.7\%) alkaloids, 12 (12.8\%) anthracycline, 10 (10.6\%) platinum compounds, 8 (8.5\%) topoisomerase inhibitors, 5 (5.3\%) alkylating agents, and 3 (3.2\%) were receiving antitumor hormonal drug therapy. In the targeted chemotherapeutic group, 11 (32.4\%) patients were using EGFR inhibitors, 11 (32.4\%) BRAF and MEK inhibitors, 7 (20.6\%) HER2/neu inhibitors, 3 (8.8\%) angiogenesis inhibitors, and 2 (5.9\%) proteasome inhibitors. Equal numbers of patients (11) were using EGFR and BRAF/ 
MEK inhibitors in the targeted chemotherapeutic group. Various mucocutaneous findings were observed at different rates in all patients. The prevalence of the common side-effects occurring is shown in Figure 1. Due to the severity of mucocutaneous side-effects, chemotherapy was stopped in 17 patients, 13 of whom were using classic chemotherapeutic agents and four of whom were using targeted chemotherapeutic agents.

The prevalence of the most common side-effects in the two groups is shown in Figure 2. According to Figure 2, except for nail changes and mucositis, all side-effects were less frequent in patients using targeted chemotherapeutics than in those using classic chemotherapeutics. No statistically significant differences were observed in terms of the most common side-effects in the classic and targeted chemotherapeutic groups. The frequencies of the common side-effects were also similar between the two groups. However, a value approaching statistical significance was determined between the two groups for skin pigmentation only ( $p=0.061 ; \chi^{2}$ test).

The findings of the present study were also grouped according to skin, hair, nail, and mucosal involvement. The most common side-effects in patients using both classic and targeted chemotherapeutics were skin changes $(94.5 \%)$, followed by changes in the hair (50\%), nails (51.6\%), and mucosa (26.6\%). The prevalence of the most common side-effects in the form of skin, skin, hair, and mucosal involvement groups is shown in Table 1.

Xerosis was the most common cutaneous side-effect, seen in $93(72.7 \%)$ patients. Other common side- effects were alopecia in 64 (50\%) patients, pruritus in 63 (49.2\%), nail changes in 66 (51,6\%), mucositis in 34 (26.6\%), skin pigmentation in $30(23.4 \%)$, and palmarplantar erythrodysesthesia in 35 (27.3\%). The least common side-effects, seen in 1 patient each, were Ara-C ears, epidermal dystrophy, and acute GVHD (Figure 2).

The most common side-effects in the classic chemotherapeutic group were xerosis seen in 71 (75.5\%) patients, pruritus in 50 (53.2\%), alopecia in 49 (52.1\%), palmar-plantar erythrodysesthesia in 42 (40.9\%), nail changes in 46 (36.8\%), skin pigmentation in 26 (27.7\%), and mucositis in 23 (24.5\%). The most common sideeffects in the targeted chemotherapeutic group were xerosis seen in 22 (64.7\%) patients, alopecia in 15 (44.1\%), pruritus in 13 (38.2\%), mucositis in 11 (32.4\%), palmarplantar erythrodysesthesia in 16 (25.6\%), nail changes in 20 (15.6\%), and skin pigmentation in 4 (11.8\%). The most common side-effects in the two groups were xerosis, pruritus, and alopecia, although the respective frequencies differed (Figure 2).

Among the specific side-effects determined in patients using classic chemotherapeutics, acute GVHD was determined in only 1 patient using vincristine among the alkaloids, while chronic GVHD was determined in 1 patient using epirubicin from the anthracyclines group, and paraneoplastic pemphigus was observed in $2 \mathrm{pa-}$ tients, one using docetaxel from the alkaloid group and the other chlorambucil, from the alkylating agents. Ara-C ears were observed in 1 patient using the antimetabolite cytosine, the chemotherapeutic-related eccrine reaction

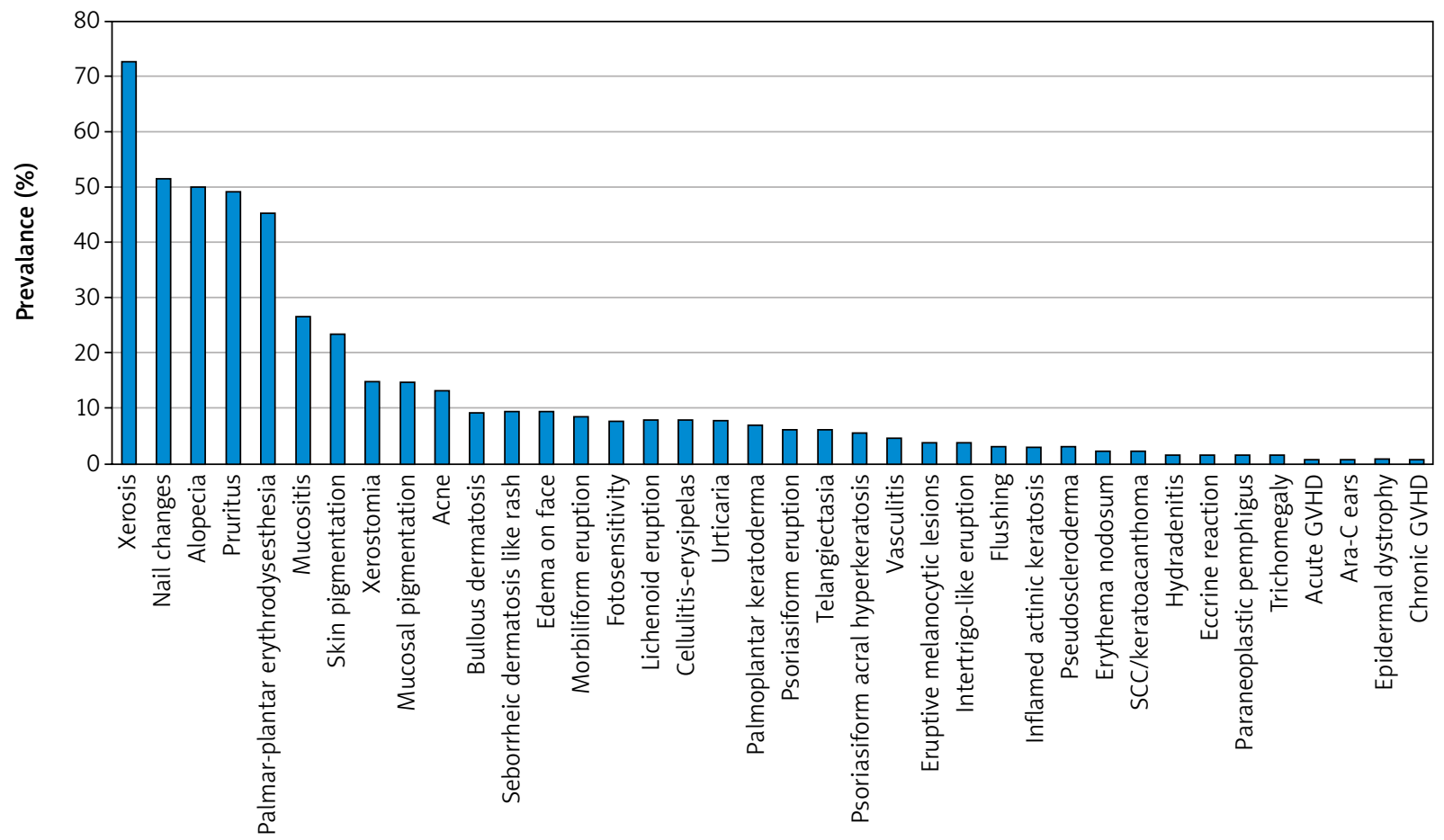

Figure 1. Prevalence of the common side-effects occurring among the entire patient group 


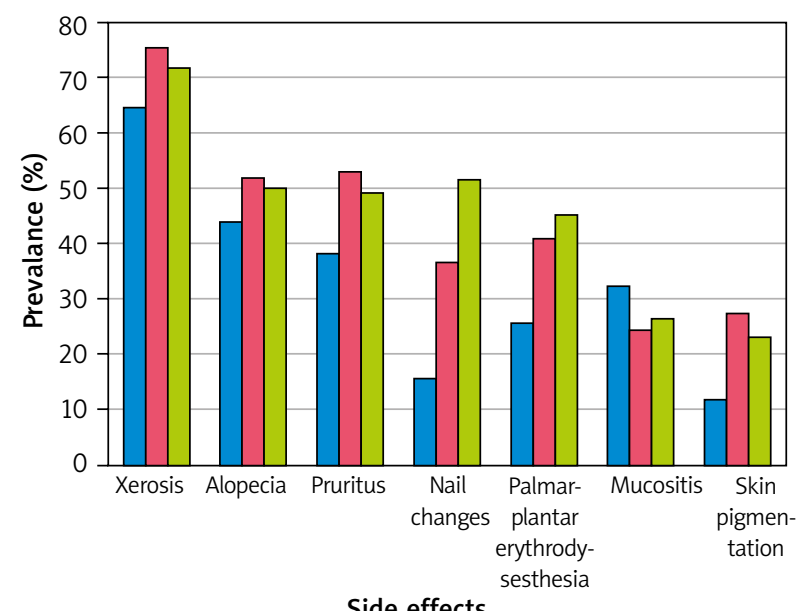

UUsing targeted chemotherapeutic group $\square$ Using classic chemotherapeutic group $\square$ Total

Figure 2. Prevalence of the most common side-effects in the two groups

Table 1. The prevalence of the most common side-effects as skin, nail, hair and mucosal involvement groups

\begin{tabular}{lccc}
\hline $\begin{array}{l}\text { Involvement } \\
\text { site }\end{array}$ & \multicolumn{3}{c}{ Patients using chemotherapeutic agents } \\
\cline { 2 - 4 } & $\begin{array}{c}\text { Classic } \\
(n=94)\end{array}$ & $\begin{array}{c}\text { Targeted } \\
(n=34)\end{array}$ & $\begin{array}{c}\text { Total } \\
(n=128)\end{array}$ \\
\hline Skin & $68.7 \%$ & $25.8 \%$ & $94.5 \%$ \\
\hline Nail & $36.8 \%$ & $15.6 \%$ & $51.6 \%$ \\
\hline Hair & $38.3 \%$ & $11.7 \%$ & $50.0 \%$ \\
\hline Mucosa & $27.3 \%$ & $10.9 \%$ & $26.6 \%$ \\
\hline
\end{tabular}

Skin - acne, ara-C ears, bullous dermatosis, cellulitis-erysipelas, eccrine re action, oedema on face, epidermal dystrophy, eruptive melanocytic lesions, erythema nodosum, flushing, photosensitivity, hidradenitis, inflamed actinic keratosis, intertrigo-like eruption, lichenoid eruption, morbiliform eruption, palmar-plantar erythrodysesthesia, palmoplantar keratoderma, pruritus, pseudoscleroderma, psoriasiform acral hyperkeratosis, psoriasiform erup tion, SCC/keratoacanthoma, seborrheic dermatosis like rash, skin pigmenta tion, telangiectasia, urticaria, vasculitis, xerosis, acute GVHD, chronic GVHD, paraneoplastic pemphigus. Hair - alopecia, trichomegaly. Nail - nail changes (discoloration, total dystrophy, pitting, subungual splinter haemorrhage). Mucosa - mucosal pigmentation, mucositis, xerostomia, acute GVHD, chronic GVHD, paraneoplastic pemphigus.

was observed in 2 patients, epidermal dystrophy was determined in 1 patient using the topoisomerase inhibitor etoposide, and trichomegaly was observed in 1 patient using the alkaloid paclitaxel. Specific side-effects among patients using targeted chemotherapeutics included chronic GVHD in 2 patients, one using nilotinib from the BRAF and MEK inhibitor group and the other using axitinib. Hidradenitis was observed in 1 patient using the EGFR inhibitor erlotinib and in two using the tyrosine kinase inhibitor imatinib, and trichomegaly was determined in 1 patient using the EGFR inhibitor cetuximab.
Table 2. The prevalence of the less common side-effects observed in the two groups

\begin{tabular}{|c|c|c|}
\hline Side-effects & $\begin{array}{l}\text { No. cases } \\
\text { (\% of total) }\end{array}$ & Drug \\
\hline \multicolumn{3}{|c|}{ Classic chemotherapeutic group $(n=94)$ : } \\
\hline Paraneoplastic pemphigus & $2(1.6 \%)$ & $\begin{array}{l}\text { Docetaxel and } \\
\text { chlorambucil }\end{array}$ \\
\hline Eccrine reaction & $2(1.6) \%$ & $\begin{array}{c}\text { Axitinib and } \\
\text { epirubicin }\end{array}$ \\
\hline Ara-C ears & $1(0.8 \%)$ & $\begin{array}{l}\text { Cytosine } \\
\text { arabinoside }\end{array}$ \\
\hline Acute GVHD & $1(0.8 \%)$ & Vincristine \\
\hline Chronic GVHD & $1(0.8 \%)$ & Epirubicin \\
\hline Epidermal dystrophy & $1(0.8 \%)$ & Etoposide \\
\hline Trichomegaly & $1(0.8 \%)$ & Paclitaxel \\
\hline \multicolumn{3}{|c|}{ Targeted chemotherapeutic group $(n=34)$ : } \\
\hline Chronic GVHD & $2(1.6 \%)$ & $\begin{array}{l}\text { Nilotinib, } \\
\text { axitinib }\end{array}$ \\
\hline Hidradenitis & $2(1.6 \%)$ & $\begin{array}{l}\text { Erlotinib and } \\
\text { imatinib }\end{array}$ \\
\hline Trichomegaly & $1(0.8 \%)$ & Cetuximab \\
\hline
\end{tabular}

The prevalence of less common side-effects in the two groups is shown in Table 2.

Chemotherapy was stopped due to the severity of mucocutaneous side-effects in 17 patients, 13 of whom were using classic chemotherapeutic agents and four of whom were using targeted chemotherapeutic agents. Classic chemotherapeutic agents caused more serious side-effects requiring discontinuation of treatment than targeted chemotherapeutic agents.

\section{Discussion}

Numerous cutaneous side-effects develop secondary to chemotherapeutics. Reported side-effects include alopecia, trichomegaly, and curly eyelashes, stomatitis (mucositis), nail changes, dry skin, neutrophilic eccrine hidradenitis, eccrine squamous syringometaplasia, acral erythema, toxic erythema, acneiform eruption, intertrigo-like eruption, hyperpigmentation, autoimmune reactions, leg ulcers, vasomotor changes, flushing, phototoxic dermatitis, radiation recall phenomenon, radiation pain flare, hypersensitivity reaction, drug extravasation, hydroxyurea dermopathy, and secondary malignancies [5]. Some of these side-effects can be easily treated, although the dosage may have to be reduced in others, or treatment may even have to be discontinued. Early identification of side-effects affects the patient's quality of life and the success of treatment [10].

Although alopecia has been reported in the literature as the most widespread dermatological adverse reaction developing in association with chemotherapeutic agents, 
increases in the frequency and severity of acral erythema, paronychia, acneiform eruption, drug extravasation, neutrophilic eccrine hidradenitis, erythema multiforme, and bullous dermatosis have been observed with the use of novel targeted agents [11].

Pavey et al. [12] evaluated mucocutaneous adverse reactions in 53 patients using both classic and targeted novel chemotherapeutics and observed nail changes in $62.2 \%$, hair changes in $37.7 \%$, cutaneous changes in $33.9 \%$, and mucosal changes in 3.7\%. A different study compared mucocutaneous reactions in 226 patients receiving chemotherapy and radiotherapy and reported nail changes in $85.84 \%$, cutaneous changes in $84.51 \%$, hair changes in $70.35 \%$, and mucosal changes in $15.04 \%$ [13]. The most common side-effects in our patients using both classic and targeted chemotherapeutics were skin changes, followed by nail, hair, and mucosal changes. Skin changes were predominant and more prevalent in the present study compared with the previous literature.

One previous study reported that the most common cutaneous side-effects were skin pigmentation (46.46\%), followed by pruritus (44.69\%), acral erythe$\mathrm{ma} /$ palmar-plantar erythrodysesthesia (27.43\%), and xerosis (20.35\%) [13]. In contrast, in the present study, xerosis $(72.7 \%)$ was the most common cutaneous sideeffect, followed by pruritus (49.2\%), skin pigmentation (23.4\%), and palmar plantar-erythema (27.3\%). Xerosis was more prevalent among patients using classic chemotherapeutics, at $75.5 \%$. Xerosis was more prevalent in platinum compounds at $90 \%$ and was observed in all patients using cisplatin and carboplatin in that group. The prevalence of xerosis in targeted treatments varied considerably in a different study, from 4\% to 35\% [14]. The equivalent figure in the present study, $64.7 \%$, was higher than in previous research. The high incidence of xerosis in both patient groups in the present study may be attributed to the cold and dry climatic conditions in the region where it was conducted $[15,16]$.

Studies have reported that hyperpigmentation may develop specific to the drug used in an average of $20 \%$ of patients [17-20]. Skin pigmentation in the present study was seen in $27.7 \%$ of the group using classic chemotherapeutics and in $11.8 \%$ of those using targeted chemotherapeutics. This difference was close to statistical significance ( $p=0.061)$. The prevalence of pigmentation in the present study was close to the values reported in the majority of previous research. One study involving 5 patients reported melasma-like pigmentation in patients using imatinib [19]. However, no melasma-like pigmentation was observed in patients using imatinib in the present study. In contrast to the previous literature, hidradenitis, one of the rare side-effects, was seen in patients using imatinib.

Alopecia was the most common side-effect in chemotherapy patients reported by Sanches et al. [20], and was also detected in approximately half (46.9\%) of our patients. Although alopecia was more common (52.1\%) in the group using classic chemotherapeutics (70\% in platinum compounds), it was also more frequently observed in the group using targeted chemotherapeutics (44.1\%) than in the previous literature (Figure 2).

Hypertrichosis is generally associated with the use of EGFR inhibitors [21]. Naveed et al. [13] reported hypertrichosis in 8 patients using imatinib, gefitinib, and temozolomide. However, it was observed in only 2 patients in the present study, and the agents responsible for it were cetuximab and paclitaxel.

Mucosal changes with chemotherapeutic use have been reported at rates of approximately $15-40 \%$ in the previous literature. Mucosal changes were detected at a rate of $26.6 \%$ in the present study, which is consistent with previous findings $[13,17]$.

The third most frequent general side-effect of chemotherapy, following alopecia and mucositis, is reported to be palmar-plantar erythema [20]. Another study reported acral erythema in $2.01 \%$ of 2186 patients [22]. In the present study, palmar-plantar erythrodysesthesia was detected in $40.9 \%$ of patients using classic chemotherapeutics, in $25.6 \%$ of those using targeted chemotherapeutics, and in $45.3 \%$ of all patients (Figure 2 ).

Although there have been several case reports concerning paraneoplastic pemphigus, one of the rarer sideeffects in the present study, the majority of cases have been linked to lymphoma and chronic myeloid leukaemia [23]. Paraneoplastic pemphigus has been reported as a side-effect of fludarabine [24]. In the present study, it was observed in 1 patient using docetaxel and in another using chlorambucil. There is no information in the literature concerning docetaxel and chlorambucil. Since the cases of paraneoplastic pemphigus observed following docetaxel and chlorambucil use in the present study represent the first reports in the literature, physicians should be aware of this side-effect, and particular care must be taken in patients scheduled to use these chemotherapeutics.

The cutaneous side-effects of newly developed targeted chemotherapeutics may not have been fully identified. Some side-effects either encountered very rarely or that had never been described in previous studies were observed in the present research. Bullous and psoriasiform dermatosis not previously reported in the literature were identified in our patient using bevacizumab, a VEGF inhibitor. In contrast to the side-effects reported for the EGFR inhibitor cetuximab, the very rare entity trichomegaly was observed in 1 patient. The side-effect profile of erlotinib is similar to that of cetuximab, although ingrowing nails, paronychia, and pyogenic granulomas can also be seen in association with erlotinib and gefitinib use [20]. Hidradenitis was also observed in 1 patient using erlotinib in the present study. Side-effects in our only patient using vandetanib included skin and mucosal pigmentation, psoriasiform eruption, and nail changes. Ac- 

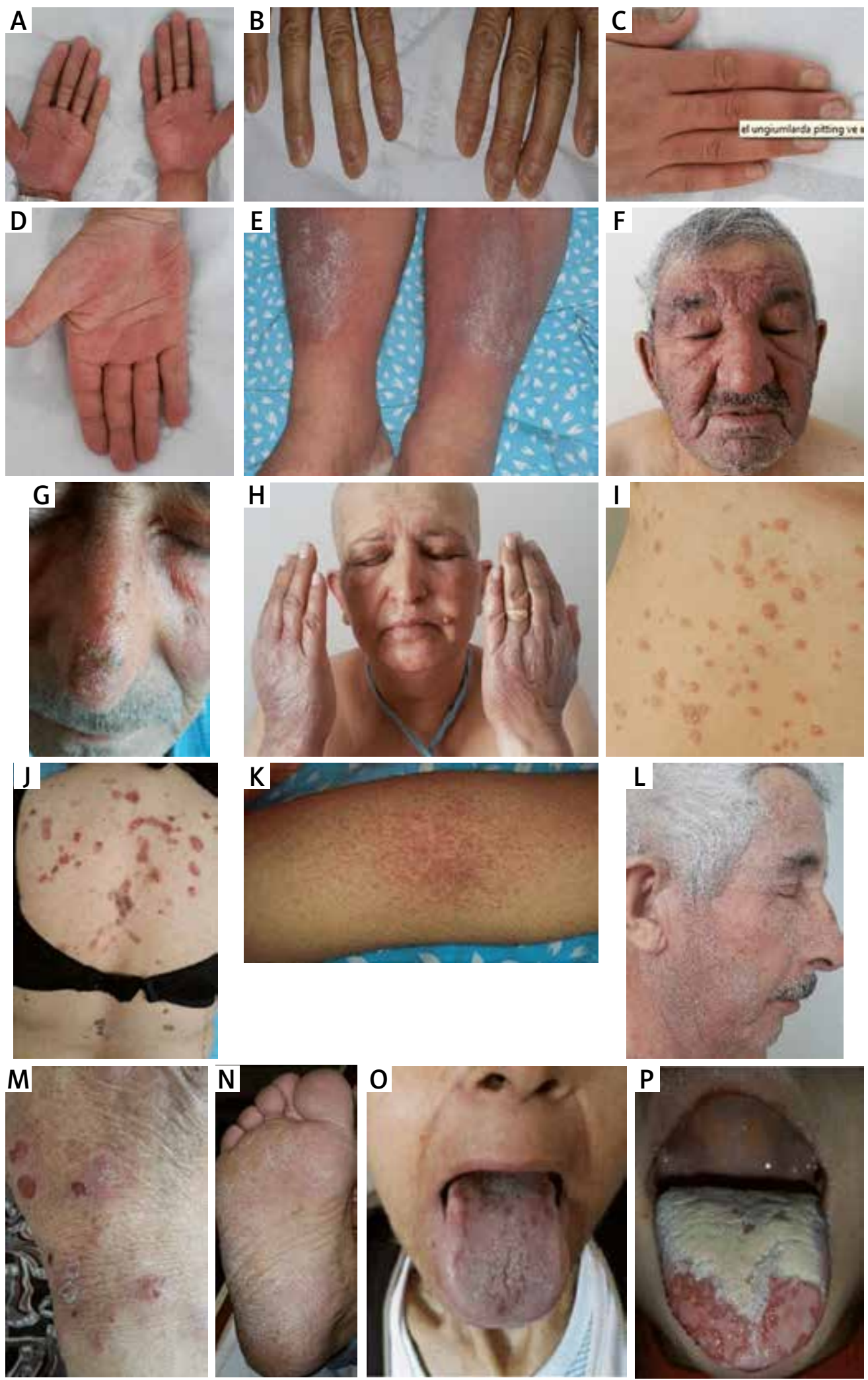

Figure 3. Various mucocutaneous findings observed in some patients: A - palmoplantar erythrodysesthesia associated with lapatinib use, B - discoloration and total dystrophy associated with 5-FU, C - pitting and discoloration associated with imatinib, D - psoriasiform plaques associated with vincristine, $\mathbf{E}$ - psoriasiform plaques associated with paclitaxel, $\mathbf{F}$ - acneiform eruption associated with cetuximab, $\mathbf{G}$ - inflamed actinic keratosis associated with axitinib, $\mathbf{H}$ - cutaneous pigmentation associated with trastuzumab, I - palmoplantar erythrodysesthesia associated with pertuzumab, J - paraneoplastic pemphigus associated with chlorambucil, $\mathbf{K}$ - lichenoid eruption associated with trastuzumab, $\mathbf{L}-$ chronic GVHD associated with nilotinib, $\mathbf{M}$ - bullous dermatosis associated with nilotinib, $\mathbf{N}$ - psoriasiform acral hyperkeratosis associated with 5-FU, $\mathbf{O}$ - mucosal pigmentation associated with sorafenib, $\mathbf{P}$ - mucositis associated with rituximab 
cording to the FDA bulletin, the side-effects of nilotinib include rash, pruritus, erythema, eczema, urticaria, alopecia, xerosis, and ecchymosis [25]. Chronic GVHD was observed in only 1 patient using nilotinib in this study. There have been previous reports on this subject, albeit few. In contrast to the previous literature, morbilliform eruption was determined in our patient using sorafenib. The HER2/neu inhibitor trastuzumab has a wide reported side-effect profile. Reported cutaneous side-effects include maculopapular eruption, erythema, pruritus, sweating, nail disorders, xerosis, alopecia, and acne [26].

Although fewer mucocutaneous side-effects were observed in the group using targeted chemotherapeutics compared to the group using classic chemotherapeutics, no statistically significant difference was observed between the two $(p<0.05)$.

Despite similarities between the side-effects observed in this study and those reported in the previous literature, we also observed side-effects that had not previously been described. However, considering the low patient numbers and the large number of subgroups investigated in this study, our findings concerning the sideeffects and their incidences between targeted and classic chemotherapeutics cannot be regarded as definitive.

The fact that xerosis was more prevalent than in other studies may be because this research was conducted in a region with a dry, cold climate. Further studies with larger patient numbers from different regions and districts are therefore needed to confirm our findings. Some photographs of skin, nail, hair, and mucosal involvements developing after the use of chemotherapeutic agents are shown in Figure 3.

\section{Conclusions}

Classic chemotherapeutics frequently give rise to mucocutaneous side-effects in cancer patients. Although newly developed targeted chemotherapeutics have begun being used, their side-effect profiles are as yet unclear. Our detection of previously unidentified side-effects possibly associated with targeted chemotherapeutics, or that may have emerged incidentally, will help guide further studies. We think that our description of new findings that have not previously been reported in this review of the potential side-effects of chemotherapeutics will help create awareness among specialists using these drugs. However, our low patient numbers represent a limitation in terms of comparing the mucocutaneous side-effects of classic and targeted chemotherapeutics, with only the most common side-effects being capable of comparison. We think that more extensive studies with larger patient populations in different regions are now needed to bring greater clarity to this subject.

\section{Conflict of interest}

The authors declare no conflict of interest.

\section{References}

1. Jemal A, Bray F, Center MM, et al. Global Cancer Statistics. CA A Cancer J Clinicians 2011; 61: 69-90.

2. Ferlay JSI, Ervik M, Dikshit R, et al. Cancer Incidence and Mortality Worldwide: IARC CancerBase No. 11. Lyon, France: International Agency for Research on Cancer. v.01; 2013.

3. TUIK. Ölüm Nedeni İstatistikleri. [cited 2014 April 15]. Available from: http://www.tuik.gov.tr/PreHaberBultenleri.do;jsessionid =2nV8h3nTghK6V7TSTSmJBY12L5cFp8 dkyXNrOSNysDtLJYvG9LpT!36722180?id=18855.

4. Payne AS, James WD, Weiss RB. Dermatologic toxicity of chemotherapeutic agents. Semin Oncol 2006; 33: 86-97.

5. Reyes-Habito CM, Roh EK. Cutaneous reactions to chemotherapeutic drugs and targeted therapies for cancer: Part I. Conventional chemotherapeutic drugs. J Am Acad Dermatol 2014; 71: 203.e1-12.

6. Frank RL. The impact of pharmaceutical innovation on premature cancer mortality in Canada, 2000-2011. Int J Health Econ Manag 2015; 15: 339-59.

7. Peuvrel L, Dréno B. Dermatological toxicity associated with targeted therapies in cancer: optimal management. Am J Clin Dermatol 2014; 15: 425-44.

8. Vaupel P. Tumor microenvironmental physiology and its implications for radiation oncology. Semin Radiat Oncol 2004; 14: 198-206.

9. Cubero DIG, Abdalla BMZ, Schoueri J, et al. Cutaneous side effects of molecularly targeted therapies for the treatment of solid tumors. Drugs Context 2018; 17: 212516.

10. Macdonald JB, Macdonald B, Golitz LE, et al. Cutaneous adverse effects of targeted therapies: Part I: inhibitors of the cellular membrane. Am Acad Dermatol 2015; 72: 203-18.

11. Wyatt AJ, Leonard GD, Sachs DL. Cutaneous reactions to chemotherapy and their management. Am J Clin Dermatol 2006; 7: 45-63.

12. Pavey RA, Kambil SM, Bhat RM. Dermatological adverse reactions to cancer chemotherapy. Indian I Dermatol Venereol Leprol 2015; 81: 434.

13. Naveed S, Thappa DV, Dubashi B, et al. Mucocutaneous adverse reactions of cancer chemotherapy and chemoradiation. Indian J Dermatol 2019; 64: 122-8.

14. Choi JN. Chemotherapy-induced iatrogenic injury of skin: new drugs and new concepts. Clin Dermatol 2011; 29: 587-601.

15. Sanborn RE, Sauer DA. Cutaneous reactions to chemotherapy: commonly seen, less described, little understood. Dermatol Clin 2008; 26: 103-19.

16. Criado PR, Brandi HR, Moure ER, et al. Adverse mucocutaneous reactions to chemotherapeutic agents: Part II. An Bras Dermatol 2010; 85: 591-608.

17. Bahçetepe N, Kutlubay Z, Pehlivan Ö, et al. Kemoterapi Ajanlarının Kutanöz Etkileri. Dermatoz 2013; 4: 69-76.

18. Rosen A, Amitay Laish I, Lacouture ME. Management algorithms for dermatologic adverse events. In: Dermatologic Principles and Practice in Oncology: Conditions of the Skin, Hair, and Nails in Cancer Patients. Mario E, Lacouture MD (eds). $1^{\text {st }}$ ed. Wiley-Blackwell, Philadelphia 2013; 365-83.

19. Ghunawat S, Sarkar R, Garg VK. Imatinib induced melasma-like pigmentation: report of five cases and review of the literature. Indian J Dermatol Vene Leprol 2016; 82: 409-12. 
20. Sanches JA, Brandt HRC, Moure ERD, et al. Adverse mucocutaneous reactions to chemotherapeutic agents: Part I. An Bras Dermatol 2010; 85: 425-37.

21. Agero AL, Dusza SW, Benvenuto AC, et al. Dermatologic side effects associated with the epidermal growth factor receptor inhibitors. J Am Acad Dermatol 2006; 55: 657-70.

22. Hueso L, Sanmartín O, Nagore E, et al. Chemotherapy-induced acral erythema: a clinical and histopathologic study of 44 cases. Actas Dermosifiliogr 2008; 99: 281-90.

23. Barnadas M, Roe E, Brunet S, et al. Therapy of paraneoplastic pemphigus with rituximab: a case report and review of the literature. J Eur Acad Dermatol Venereol 2006; 20: 69-74.

24. Gooptu CLT, Frith P, Lyon CC, et al. Paraneoplastic pemphigus: an association with fludarabine? Br J Dermatol 2001; 144: 1255-61.

25. Tasigna FDA. Highlights of prescribing information. Adverse reactions. [cited 2010 June 15]. Available from: https://www.accessdata.fda.gov/drugsatfda_docs/ label/2010/022068s004s005lbl.pdf

26. Cortazar P. Center for drug evaluation and research. Cross-discipline team leader review. Application Number: 125427orig1s000. Ado Trastuzumab emtansine KADCYLA, BLA 125427, February 19, 2013. 